\title{
Assessment of Oral Health-related Quality of Life among Patients Who have Undergone Orthodontic Treatment in Navi Mumbai
}

\author{
Samridhi Vyas ${ }^{1}$, Madhura Pednekar ${ }^{2}$, Vaibhav Thakkar ${ }^{3}$, Divij Joshi ${ }^{4}$, Sabita Ram ${ }^{5}$, Rachna Darak ${ }^{6}$, Prathamesh Fulsundar ${ }^{7}$
}

\begin{abstract}
Aim and objective: The study aimed to assess the oral health-related quality of life (OHRQoL) among patients who have undergone orthodontic treatment at a dental institute in Navi Mumbai, Maharashtra.

Materials and methods: A cross-sectional descriptive survey was carried out composed of two sets of OHIP-14 [Oral Health Impact Profile] questionnaires, former during the first month of treatment and the latter after the termination of the said treatment. In total 153 individuals between the age group of 15-30 years old who were eligible and met the inclusion and exclusion criteria from a dental institute in Navi Mumbai from June 2017 to May 2018 participated in the study.

Results: All fields of the OHIP-14 questionnaire namely functional limitations, physical pain, psychological discomfort, physical disabilities, psychological disabilities, social disabilities, and handicapped showed a highly significant decrease in the OHRQoL score post orthodontic therapy $[p<0.001]$.The highest decline with $78.45 \%$ of individuals was noted in the field of physical pain.

Conclusion: Orthodontic treatment can have a great positive influence on the quality of life. Although, orthodontic treatment might be associated with some problems and discomforts at the beginning and during the procedure but by the completion of the treatment, all of the quality of life domains show improvement.

Clinical significance: This information from this study can be used for "informed consent," which may increase patients' cooperation as they are aware of what is to be expected during orthodontic treatment.

Keywords: OHRQoL, Oral health-related quality of life, Orthodontic treatment, Questionnaire study.

World Journal of Dentistry (2022): 10.5005/jp-journals-10015-1908
\end{abstract}

\section{INTRODUCTION}

Malocclusion is one of the most common developmental anomalies which usually manifests itself during childhood either due to malalignment of teeth or an abnormal relation of the dental arches. ${ }^{1,2}$ It leads to several issues like problems associated with the function of the masticatory system, dysfunction of the temporomandibular joint (TMJ), problems with swallowing and speech, susceptibility to facial traumatic injuries, and development of caries and periodontal problems. ${ }^{3}$

Besides, the individuals with malocclusion will not be satisfied with their facial appearance, resulting in inappropriate social responses, and development of emotional and mental problems. ${ }^{1,4}$

Considering the established relationship between aesthetic, health, and satisfaction with an individual's appearance and social function, malocclusion might result in a decrease in self-confidence and individual's social functioning, especially in adolescents. ${ }^{5}$

Investigators prefer to use the change of cephalometric planes and angles or peer assessment rating scores before and after orthodontic treatment as outcome measures. However, the recommendation by World Health Organization that quality of life $(\mathrm{QoL})$ measures should be included in clinical studies has resulted in more emphasis on inclusion of patient- centred outcome measures when studying orthodontic treatments and outcomes. ${ }^{6}$

O'Brien et al. ${ }^{7}$ emphasized that while clinical indicators of treatment outcomes are still important, oral health-related QoL (OHRQoL) measures that take into account these broader health concepts are important, especially since patient-oriented
${ }^{1}$ Private Practitioner, Mumbai, Maharashtra, India

${ }^{2}$ Department of Orthodontics and Dentofacial Orthopaedics, MGM Dental College and Hospital, Navi Mumbai, Maharashtra, India

${ }^{3}$ Department of Public Health Dentistry, MGM Dental College and Hospital, Navi Mumbai, Maharashtra, India

${ }^{4}$ Department of Orthodontics and Dentofacial Orthopaedics, Bharati Vidyapeeth Dental College and Hospital, Navi Mumbai, Maharashtra, India

${ }^{5}$ MGMIHS, Navi Mumbai, Maharashtra, India

${ }^{6}$ Department of Orthodontics and Dentofacial Orthopaedics, Dr DY Patil Dental College and hospital, Pimpri Pune, Maharashtra, India

${ }^{7}$ Department of Prosthodontics and Crown and Bridge, Bharti Vidyapeeth Dental College and Hospital, Pune, Maharashtra, India

Corresponding Address: Madhura Pednekar, Department of Orthodontics and Dentofacial Orthopaedics, MGM Dental College and Hospital, Navi Mumbai, Maharashtra, India, Phone: +91 9920744907, e-mail: ptcasesbackup@gmail.com

How to cite this article: Vyas S, Pednekar M, Thakkar V, et al. Assessment of Oral Health-related Quality of Life among Patients Who have Undergone Orthodontic Treatment in Navi Mumbai. World J Dent 2022;13(2):161-165.

Source of support: Nil

Conflict of interest: None

OHRQoL outcomes do not necessarily correlate with objective clinical findings. ${ }^{7}$ Therefore, they insist that self-reported OHRQoL 
instruments should be applied in the assessment of orthodontic treatment because they reflect the patient's views and feelings as a supplement to clinical indices. Not only should research studies use OHRQoL in measuring the effectiveness of specific treatments but routine use of $O H R Q D L$ in daily practice could help the orthodontist not only better the diagnose and treat malocclusion but also better understand the concerns from the patient's perspective. ${ }^{6}$

Therefore, the quality of life as related to oral health in patients who have undergone fixed orthodontic treatment will be evaluated to shed more light on the effect of orthodontic treatment on OHRQoL specifically in Navi Mumbai population since such a study has not been carried out in Navi Mumbai population earlier. Thus the aim of our study is to evaluate quality of life as related to oral health in patients during and after undergoing fixed orthodontic treatment.

\section{Materials and Methods}

A cross-sectional descriptive study was conducted among 153 study participants of age group between 15 and 30 years old chosen by convenience sampling method who had undergone fixed orthodontic treatment from MGM Dental College and Hospital in Navi Mumbai, Maharashtra, India. The study protocol was approved by the Ethical Committee of the said institute with approval number MGM/DCH/IERC/20/18. The nature of the study was explained to each study participant and written informed consent was obtained. Data was obtained by two examiners using two sets of the OHIP-14 [Oral Health Impact Profile] questionnaire, ${ }^{8}$ former during the first month of treatment and the latter after the termination of the said treatment. OHIP-14 questionnaire is a prevalidated questionnaire. The English questionnaire was additionally translated in the local Hindi and Marathi language. It consisted of 14 questions, measuring the quality of life in the seven fields of functional limitations, physical pain, psychological discomfort, physical disabilities, psychological disabilities, social disabilities, and handicapped.

\section{Inclusion Criteria}

Individuals within the age group of 15-30 years who had undergone orthodontic treatment and who were willing to give consent to participate in the study.

\section{Exclusion Criteria}

Individuals who were not willing to take part in the study.

Individuals with a history of maxillofacial surgeries, any systemic or mental problems, and any manifested disorders in the general growth pattern.

The study participant answered each of these concerned questions and explained his/ her experience with the problem arising from the teeth and the oral condition during the past 12 months.

The study participant's answers were scored in the LICKERT'S SCALE as zero for "never," 1 for "seldom," 2 for "sometimes," 3 for "mostly," and 4 for "almost always."

On the whole, a score ranging between " 0 " and " 56 " is calculated for each study participant and each domain scores can range from 0 to 8 . Higher scores indicated a lower quality of life for the subjects.
In the present study, in the final evaluation of answers, the "zero" response would be considered a lack of effect and answers 1-4 will be considered an effect so that the comparisons would be more comprehensible.

The Statistical Package for the Social Sciences [version 24.0; SPSS Inc, Chicago, IL, USA] was used for the data analysis. Descriptive analyses were performed with frequency distribution and cross-tabulation. The Kolmogorov-Smirnov test was used to test the normality of the sample. The Chi-square was used to test associations between categorical data. The significance of all the tests was predetermined at the probability value of 0.05 or less.

\section{Results}

The mean age of the study participants was $20.40 \pm 4.242$ years. (Table 1) The 153 patients who had undergone orthodontic treatment between June 2017 to May 2018 were selected.

The OHIP-14 [Oral Health Impact Profile] questionnaires were used to find out about the quality of life in the Seven Fields of Functional Limitations, Physical Pain, Psychological Discomfort, Physical Disabilities, Psychological Disabilities, Social Disabilities, and Handicapped. ${ }^{8}$

\section{Functional Limitations}

OHIP-1 and 2.

This included questions regarding the limitations in pronouncing the words and the sensing of the taste of food.

\section{Physical Pain}

OHIP-3 and 4

These questions consisted of discomfort while eating and the painful aching experienced by the study participants.

\section{Psychological Discomfort}

\section{OHIP-5, 6, and 10}

These sets of questions included the psychological discomfort i.e., being self-conscious, being tensed and embarrassed by the treatment.

\section{Physical Disabilities}

OHIP-7, 8, and 14

This domain included questions regarding the physical disability experienced which included unsatisfactory diet, interruption of meals and inability to function.

\section{Psychological Disabilities}

OHIP-9

This question consisted of the difficulty to relax by the study participant.

\section{Social Disabilities}

OHIP-11 and 12

These questions were based on the understanding of the treatment affecting the occupation of the study participant and the level of irritability experienced.

Table 1: Demographic characteristics of the study participants $(N=153)$

\begin{tabular}{llll}
\hline Variables & Subgroups & $N$ & Percentage \\
\hline Gender & Female & 94 & 61.4 \\
& Male & 59 & 38.6 \\
\hline
\end{tabular}




\section{Handicapped}

\section{OHIP - 13}

This question was to know if the study participant felt his life less satisfactory overall due to the treatment.

This was the result of 153 study participants post orthodontic treatment (Table 2), (Fig. 1) $64.7 \%$ of study participants showed significant improvement involving functional limitation, $78.45 \%$ of study participants showed considerable decline in physical pain, $62.93 \%$ of study participants showed substantial fall in psychological discomfort, $47.7 \%$ of study participants showed marginal improvement involving physical disabilities, 70.60\% of study participants showed a great decline in psychological disabilities, $52.30 \%$ of study participants showed a great advance involving social disabilities, $34.60 \%$ of study participants showed borderline satisfaction from life, $63.40 \%$ of study participants showed a neutral response for life satisfaction post treatment.

\section{Discussion}

This study is a longitudinal study and it better quantifies the change in health status and provides better evidence on treatment effects. ${ }^{9}$ In current times, the measurement of OHRQoL is an essential component of oral health surveys, clinical trials, and studies evaluating the outcomes of preventive and therapeutic programs intended to improve oral health. The assessment of the oral health-related quality of life is an important tool in clinical practice $^{10}$ and is invaluable in the practice of orthodontics as orthodontic problems and their respective corrections have a large psychosocial impact. Orthodontic treatment has shown to have a statistically significant impact on OHRQoL. Our finding that OHRQoL was negatively affected during orthodontic treatment has been reported previously. ${ }^{11,12}$ In our research, total study participants included were 153 ( 94 females and 59 males).

A highly significant difference is seen in attributes of functional limitations i.e., pronunciation of words and sensation of taste during and after treatment. This could result due to the free movement of lips and tongue. This finding coincided with that by Liu et al. ${ }^{17}$ and Costa et al. ${ }^{13}$ The second variable of physical pain which included questions about discomfort while eating and the painful aching experienced by the study participants showed decrease in OHRQoL score in our study, similar to study by Kang and Kang. ${ }^{14}$ This could be attributed to absence of fixed appliance in the posttreatment stage thus facilitating ease of eating, lack of food lodgment in the appliance, release of pressure, stasis of tooth movement. Psychological discomfort domain involving questions of being self-conscious, being tensed and embarrassed by the treatment also showed a highly significant decrease during and after treatment. An explanation for the decrease

Table 2: Comparison of the responses to the questions in terms of [Mean (SD)] before and after the treatment using Wilcoxon Signed rank test

\begin{tabular}{|c|c|c|c|c|c|c|}
\hline Variables & Time interval & $N$ & Mean & Std. Deviation & Zvalue & $p$-value \\
\hline \multirow{2}{*}{ Problem with words? } & During & 153 & 1.93 & 0.918 & 9.220 & $<0.001^{b}$ \\
\hline & Post & 153 & 0.77 & 0.721 & & \\
\hline \multirow{2}{*}{ Sense of taste worsened? } & During & 153 & 1.38 & 1.051 & 8.064 & $<0.001^{\mathrm{b}}$ \\
\hline & Post & 153 & 0.54 & 0.659 & & \\
\hline \multirow{2}{*}{ Painful ache? } & During & 153 & 2.65 & 1.041 & 9.736 & $<0.001^{\mathrm{b}}$ \\
\hline & Post & 153 & 0.88 & 0.789 & & \\
\hline \multirow{2}{*}{ Uncomfortable to eat food? } & During & 153 & 2.14 & 0.976 & 8.754 & $<0.001^{\mathrm{b}}$ \\
\hline & Post & 153 & 0.84 & 0.812 & & \\
\hline \multirow{2}{*}{ Been conscious? } & During & 153 & 1.64 & 1.201 & 7.641 & $<0.001^{b}$ \\
\hline & Post & 153 & 0.73 & 0.868 & & \\
\hline \multirow{2}{*}{ Felt tense? } & During & 153 & 1.31 & 1.154 & 7.413 & $<0.001^{\mathrm{b}}$ \\
\hline & Post & 153 & 0.52 & 0.779 & & \\
\hline \multirow{2}{*}{ Unsatisfactory diet? } & During & 153 & 2.22 & 1.181 & 9.002 & $<0.001^{b}$ \\
\hline & Post & 153 & 0.67 & 0.724 & & \\
\hline \multirow{2}{*}{ Interrupt meals? } & During & 153 & 1.95 & 1.037 & 9.137 & $<0.001^{b}$ \\
\hline & Post & 153 & 0.56 & 0.751 & & \\
\hline \multirow{2}{*}{ Difficult to relax? } & During & 153 & 1.61 & 0.947 & 8.951 & $<0.001^{\mathrm{b}}$ \\
\hline & Post & 153 & 0.53 & 0.717 & & \\
\hline \multirow{2}{*}{ Been a bit embarrassed? } & During & 153 & 1.88 & 1.169 & 8.287 & $<0.001^{b}$ \\
\hline & Post & 153 & 0.76 & 0.803 & & \\
\hline \multirow{2}{*}{ Irritable with people? } & During & 153 & 1.01 & 1.100 & 6.171 & $<0.001^{\mathrm{b}}$ \\
\hline & Post & 153 & 0.48 & 0.699 & & \\
\hline \multirow{2}{*}{$\begin{array}{l}\text { Difficulty in doing the usual } \\
\text { jobs? }\end{array}$} & During & 153 & 0.93 & 1.011 & 6.217 & $<0.001^{\mathrm{b}}$ \\
\hline & Post & 153 & 0.33 & 0.583 & & \\
\hline \multirow{2}{*}{ Life less satisfying? } & During & 153 & 0.70 & 1.027 & 6.075 & $<0.001^{\mathrm{b}}$ \\
\hline & Post & 153 & 0.16 & 0.567 & & \\
\hline \multirow{2}{*}{ Unable to function? } & During & 153 & 0.63 & 0.817 & 6.887 & $<0.001^{b}$ \\
\hline & Post & 153 & 0.07 & 0.283 & & \\
\hline
\end{tabular}

$\left(p<0.05\right.$ - Significant ${ }^{\mathrm{a}}, p<0.001$ - Highly significant $\left.{ }^{\mathrm{b}}\right)$ 


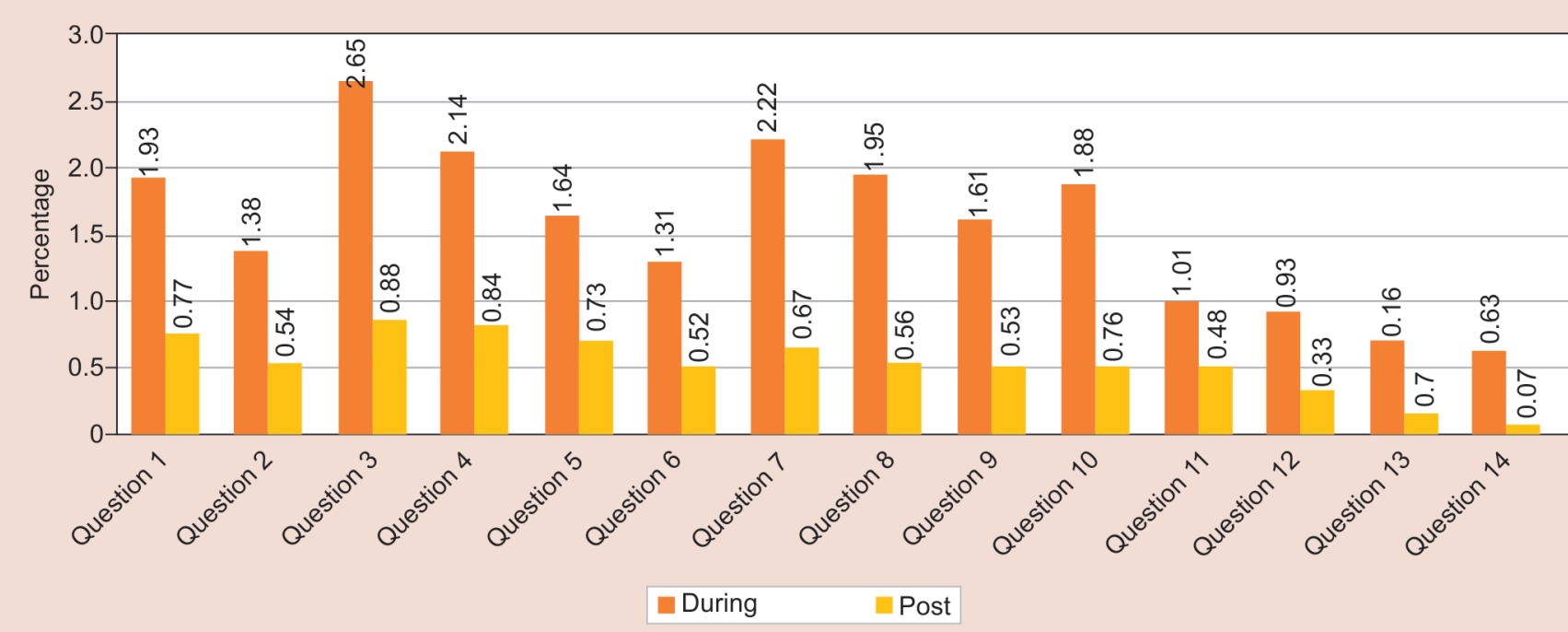

Fig. 1: Graph depicting change in OHRQoL variables during and post orthodontic treatment

could be that study participants see their teeth move to a better, more aesthetic position after the completion of treatment. The fourth domain of physical disabilities consisting of questions regarding unsatisfactory diet, interruption of meals and inability to function showed a reduction in scores posttreatment. This could be attributed to the prohibition of eating hard, sticky and chewy foods during treatment. ${ }^{15}$ Also, patients are unable to masticate permitted foodstuffs due to pain on occluding. ${ }^{16}$ The psychological disability showed reduced scores posttreatment and increased ability of study participants to relax posttreatment which can be due to alleviation of pain and pressure sensation. Social disability questions based on the understanding of the treatment affecting the occupation of the study participant and the level of irritability experienced showed a significant reduction in scores. This could be due to decreased pain and not missing workdays due to orthodontic appointments, etc. The last domain of Handicap to know if the study participant felt his/ her life less satisfactory overall due to the treatment showed a significant reduction posttreatment. Thus, our study showed amelioration in OHRQoL during and posttreatment similar to studies by Palomares ${ }^{17}$, Fahimeh. ${ }^{18-23}$

Some limitations of our study have to be considered. First, OHRQoL is a subjective evaluation of a study participant's own experience and perception. A balanced distribution of either gender should have been done as it would signify the difference of perceptions as it was reported by Kang and Kang that women show higher OHRQoL scores than men. ${ }^{14}$ This was a generalized study and no segregation was done among different treatment modalities like fixed or removable or orthopedic appliance therapy/ labial or lingual or aesthetic braces. The age group was limited to 15-30 years, but adults above the mentioned age could have been included as now many adults desire orthodontic treatment with the advent of aesthetic braces and knowing their perception will be useful for clinical practice.

Further studies emphasizing on pretreatment, various stages of treatment and posttreatment scores are recommended. The difference in perceptions of various treatment modalities like labial, lingual, aesthetic, self-ligating, aesthetic self-ligating, and aligner can be undertaken. OHRQoL between groups with and without Temporary Anchorage Devices for treatment can be carried out. There is a need to determine whether observed benefits in OHRQoL are short or long-term in nature and whether specific types or severities of malocclusion are more likely to benefit than others.

Considering the effects of wearing an orthodontic appliance on OHRQoL, orthodontists need to explain the possible discomfort and consequences of treatment. It is important to make clear that most of the negative consequences are temporary and get better during or after the treatment. ${ }^{17}$ This information may enhance adherence to treatment, as successful orthodontics is facilitated by adequate communication between the orthodontist and patient. ${ }^{13}$

\section{Conclusion}

Orthodontic treatment provided during the study age group 15-30 years leads to improvements in OHRQoL following treatment. This resultant inference can be used while obtaining "informed consent," which may increase patients' cooperation as they become aware of what is to be expected during orthodontic treatment.

\section{Clinical Significance}

This study thus signifies the role of orthodontists in conducting counselling sessions with patients before and during the treatment to instil a positive attitude and ensure that the treatment-related problems are temporary and will cease to bother the patient once treatment is completed.

\section{References}

1. Zou J, Meng M, Law CS, et al. Common dental diseases in children and malocclusion. Int J Oral Sci. 2018;10(1): 7. Doi: 10.1038/ s41368-018-0012-3

2. CapelozzaFilho L, Braga AS, Cavassan AO, et al. Orthodontic treatment in adults: an objective approach. Rev Dent Press Ortodon Ortop Facial 2001;6:63-80. DOI: 10.1590/S2176-94512012000500008

3. Dimberg L, Arnrup K, Bondemark L. The impact of malocclusion on the quality of life among children and adolescents: a systematic review of quantitative studies. Eur J Orthod 2015;37(3):238-247. DOI: 10.1093/ejo/cju046 
4. Gazit-Rappaport T, Haisraeli-Shalish M, Gazit E. Psychosocial reward of orthodontic treatment in adult patients. Eur J Orthod 2010; 32:441-446. DOI:10.1093/ejo/cjp144

5. Gkantidis N, Christou P, Topouzelis N. The orthodontic periodontic interrelationship in integrated treatment challenges: a systematic review. J Oral Rehabil 2010;37:377-390. DOI: 10.1111/j.1365-28 42.2010.02068.x

6. Johal A, Alyaqoobi I, Patel R, et al. The impact of orthodontic treatment on quality of life and self- esteem in adult patients. Eur J Orthod 2015;37:233-237. DOI: 10.1093/ejo/cju047

7. O'Brien K, Kay L, Fox D, Mandall N. Assessing oral health outcomes for orthodontics - Measuring health status and quality of life. Community Dent Health 1998;15:22-26.

8. Slade GD, Spencer AJ: Development and evaluation of the oral health impact profile. Community Dent Health 1994,11: 3-11. DOI: 10.1007/978-94-007-0753-5_2019

9. Locker $D$, Issues in measuring change in self-perceived oral health status. Community Dent Oral Epidemiology; 26:41-47.

10. Locker D, Matear D, Stephens M, et al. Comparison of the GOHAI and OHIP-14 as measures of the oral health-related quality of life of the elderly. Community Dent Oral Epidemiol 2001; 29:373-381. DOI: 10.1034/j.1600-0528.2001.290507.x

11. Brosens V, Ghijselings I, Lemiere J, et al. Changes in oral health-related quality of life reports in children during orthodontic treatment and the possible role of self-esteem: a follow-up study. Eur Orthos 2014; 36, 186-191. DOI: 10.1093/ejo/cjt035

12. Chen M, Wang DW, Wu LP. Fixed orthodontic appliance therapy and its impact on oral health-related quality of life in Chinese patients. Angle Orthodontist 2010;80,49-53. DOI: 10.2319/010509-9.1

13. Costa AA, Serra-Negra JM, Bendo CB, et al. Impact of Orthodontics on Quality of Life. Angle Orthodontist 2016;86(1):121-126 DOI: $10.2319 / 100514716.1$
14. Kang JM, Kang KH. Effect of malocclusion or orthodontic treatment on oral health-related quality of life in adults. Korean J Orthod 2014;44(6):304-311. DOI: 10.4041/kjod.2014.44.6.304

15. Sharma R, Mittal S, Singla A, et al. Nutritional guidelines for orthodontic patients. Internet J Nutr Wellness 2009;10:1-4.

16. Khatri JM, Kolhe VD. Nutrition and orthodontics. Int J Orthod Rehabil 2018;9:163-167. DOI: 10.4103/ijor.ijor_21_18

17. Palomares N, Celeste R, de Oliverira, B et al. How does orthodontic treatment affect young adults' oral health-related quality of life? Am Ortho Dentofacial Orthop 2012;141(6),751-758. DOI: 10.1016/j. ajodo.2012.01.015

18. Fahimeh F, Farzin H, Behrad T, et al. Changes of oral health related quality of life during and after fixed orthodontic treatment. Adv Dent \& Oral Health 2016;2(4): 555598. DOI: 10.19080/ADOH.2016.02.555598

19. Azuma S, Kohzuki M, Saeki S, et al. Beneficial effects of orthodontic treatment on quality of life in patients with malocclusion, Tohoku. J Exp Med 2008; 214(1):39-50.

20. Kok YV, Mageson P, Harradine NW, et al. Comparing a quality of life measure and the Aesthetic Component of the Index of Orthodontic Treatment Need (IOTN) in assessing orthodontic treatment need and concern J Orthod . 2004; 31(4):312-318; discussion 300-1.

21. Kiyak HA. Does orthodontic treatment affect patients' quality of life? J Dent Educ 2008; 72(8):886-894.

22. Taylor KR, Kiyak A, Huang GJ, et al. Effects of malocclusion and its treatment on the quality of life of adolescents. Am J Orthod Dento facial Orthop 2009;136(3):382-392. DOI: 10.1016/j. ajodo.2008.04.022

23. Tai-Ting Lai, Jeng-Yuan Chiou, Tai-Cheng Lai, et al. Oral health-related quality of life in orthodontic patients during initial therapy with conventional brackets or self-ligating brackets. J Dent Sci 12(2):161-172. DOI: 10.1016/j.jds.2016.12.003 